\title{
25. CARBON AND CARBONATE ANALYSES, LEG 30
}

\author{
Donald H. Cameron, Scripps Institution of Oceanography, La Jolla, California
}

Leg 30 carbonate samples were collected onboard ship in 3-cc vials at the time the core was split open. On shore the samples were dried and ground to a homogeneous powder. The ground sediment was redried at $105^{\circ}-110^{\circ} \mathrm{C}$ and two samples from each vial, a $0.1-\mathrm{g}$ and a $0.5-\mathrm{g}$ sample, were then weighed into LECO crucibles. The 0.5-g sample was acidified with dilute hydrochloric acid, washed with distilled water, redried, and analyzed for acid insoluble (organic) carbon using a LECO 70 carbon analyzer (Boyce and Bode, 1972). The 0.1 -g sample was treated only with distilled water to cake the sample before analysis for total carbon. If the result showed less than $10 \% \mathrm{CaCO}_{3}$, an additional $0.5-\mathrm{g}$ sample was analyzed for greater accuracy. The calcium carbonate percentages were calculated as follows: (\% total $\mathrm{C}-\%$ organic C) $\times 8.33=\% \mathrm{CaCO}_{3}$. Although other carbonates may be present, all acid-soluble carbon was calculated as calcium carbonate. All results are given in weight percent.

Detailed descriptions of technique and theory are in the Initial Reports of the Deep Sea Drilling Project, Volume 4 (Bader, Gerard, et al., 1970).

For control purposes a standard sediment was made up from Deep Sea Drilling material and analyzed for total carbon at predetermined intervals with the regular samples. Listed below is the statistical data for this standard.

\begin{tabular}{ccccc} 
DSDP Std. & $\begin{array}{c}\text { No. of } \\
\text { Samples }\end{array}$ & $\begin{array}{c}\text { Total Carbon } \\
\text { as \% } \mathrm{CaCO}_{3}\end{array}$ & $\begin{array}{c}\text { Standard } \\
\text { Deviation }\end{array}$ & $\begin{array}{c}\text { Maximum } \\
\text { Range }\end{array}$ \\
\hline 2 & 18 & 79.6 & $0.6 \%$ & $2.1 \%$
\end{tabular}

These data indicate the precision of the mechanical aspect of the LECO analysis and do not necessarily reflect the precision of the total analytical procedure, which may be affected by factors such as sample homogeneity or contamination during sample preparation.

\section{REFERENCES}

Bader, R.G., Gerard, R.D., et al., 1970. Initial Reports of the Deep Sea Drilling Project, Volume 4: Washington (U.S. Government Printing Office).

Boyce, R.E. and Bode, G.W., 1972. Carbon and carbonate analyses, Leg 9, Deep Sea Drilling Project. In Hays, J.D. et al., Initial Reports of the Deep Sea Drilling Project, Volume 9: Washington (U.S. Government Printing Office), p. 797.
TABLE 1

Carbon-Carbonate Analyses, Leg 30

\begin{tabular}{lcccc}
\hline $\begin{array}{c}\text { Sample } \\
\text { (Interval in cm) }\end{array}$ & $\begin{array}{c}\text { Subbottom } \\
\text { Depth } \\
(\mathrm{m})\end{array}$ & $\begin{array}{c}\text { Total } \\
\text { Carbon } \\
(\%)\end{array}$ & $\begin{array}{c}\text { Organic } \\
\text { Carbon }\end{array}$ & $\mathrm{CaCO}_{3}$ \\
\hline Hole 285 & & & & \\
2-1, 138 & 18.4 & 9.1 & 0.1 & 75 \\
$2-3,86$ & 20.9 & 7.5 & 0.1 & 62 \\
$2-4,61$ & 22.1 & 9.1 & 0.0 & 75 \\
$2-5,131$ & 24.3 & 7.8 & 0.1 & 64 \\
$3-2,90$ & 38.7 & 7.8 & 0.1 & 64 \\
$3-5,60$ & 42.9 & 7.8 & 0.1 & 64 \\
$4-1,107$ & 56.1 & 6.5 & 0.1 & 54 \\
$4-4,50$ & 60.0 & 5.3 & 0.1 & 44 \\
$4-6,40$ & 62.9 & 2.0 & 0.1 & 16 \\
$5-4,80$ & 79.8 & 1.8 & 0.1 & 14 \\
& & & &
\end{tabular}

Hole 285A

$1-2,64$

133.1

5-2, 81

455.3

1.4

0.8

0.1

0.1

11

Site $\mathbf{2 8 6}$

1-4, 20

2-2, 68

$3-4,100$

$5-5,100$

$6-4,110$

$8-1,100$

9-5, 66

$15-1,140$

$17-5,63$

23-2, 95

$25-4,12$

25-4, 55

29-5, 99

$35-1,111$

4.7

18.7

41.0

80.5

98.1

131.5

156.2

264.9

308.1

418.0

458.1

458.6

536.5

644.6

\section{7}

$$
0.3
$$

1.9

Site 287

$1-3,115$
$5-4,59$
$5-4,67$
$6-3,111$
$6-3,122$
$6-3,129$
$8-1,19$
$8-1,44$
$8-3,74$
$9.2,74$
$10-1,145$
$10-2,15$
$10-2,75$
$10-3,58$
$10-4,92$
$11-4,61$
$14-3,5$

4.2
79.9
80.0
97.6
97.7
97.8
131.7
131.9
135.2
152.7
171.0
171.2
171.8
173.1
174.9
184.4
210.6

\begin{tabular}{rlr}
2.0 & 0.5 & 13 \\
5.4 & 0.2 & 43 \\
4.8 & 0.2 & 38 \\
0.9 & 0.3 & 5 \\
4.0 & 0.4 & 30 \\
4.3 & 0.4 & 32 \\
0.3 & 0.2 & 1 \\
0.2 & 0.2 & 0 \\
0.1 & 0.1 & 0 \\
0.1 & 0.1 & 0 \\
0.2 & 0.1 & 1 \\
0.1 & 0.1 & 0 \\
9.7 & 0.0 & 81 \\
9.2 & 0.1 & 76 \\
10.3 & 0.1 & 85 \\
6.8 & 0.1 & 56 \\
6.8 & 0.1 & 56 \\
\hline
\end{tabular}


TABLE 1 - Continued

\begin{tabular}{|c|c|c|c|c|}
\hline $\begin{array}{c}\text { Sample } \\
\text { (Interval in } \mathrm{cm} \text { ) }\end{array}$ & $\begin{array}{l}\text { Subbottom } \\
\text { Depth } \\
\text { (m) }\end{array}$ & $\begin{array}{c}\text { Total } \\
\text { Carbon } \\
(\%)\end{array}$ & $\begin{array}{l}\text { Organic } \\
\text { Carbon }\end{array}$ & $\mathrm{CaCO}_{3}$ \\
\hline \multicolumn{5}{|l|}{ Hole $288 \mathrm{~A}$} \\
\hline $\begin{array}{l}1-2,50 \\
3-6,53 \\
3-6,26 \\
5-3,85 \\
6-2,100 \\
9-1,41\end{array}$ & $\begin{array}{r}2.0 \\
37.3 \\
37.1 \\
71.4 \\
88.7 \\
171.9\end{array}$ & $\begin{array}{r}9.2 \\
11.1 \\
8.1 \\
9.3 \\
11.4 \\
11.3\end{array}$ & $\begin{array}{l}0.1 \\
0.1 \\
0.1 \\
0.1 \\
0.0 \\
0.1\end{array}$ & $\begin{array}{l}76 \\
92 \\
66 \\
77 \\
95 \\
93\end{array}$ \\
\hline \multicolumn{5}{|l|}{ Hole 288A } \\
\hline $\begin{array}{l}3-2,132 \\
6-1,71 \\
8-2,63 \\
8-2,144 \\
9-3,20 \\
10-1,86 \\
14-1,106 \\
16-1,99 \\
20-2,71 \\
21-2,111 \\
22-2,141 \\
23-3,99 \\
25-1,107 \\
27-2,69 \\
28-1,125 \\
29-1,114 \\
30-1,102\end{array}$ & $\begin{array}{l}345.8 \\
457.7 \\
535.1 \\
535.9 \\
574.6 \\
609.9 \\
724.1 \\
762.0 \\
848.7 \\
858.6 \\
868,4 \\
879.0 \\
895.1 \\
934.2 \\
952.3 \\
971.1 \\
980.5\end{array}$ & $\begin{array}{r}11.4 \\
11.7 \\
11.8 \\
11.7 \\
11.7 \\
11.5 \\
10.6 \\
8.3 \\
10.0 \\
9.7 \\
5.5 \\
8.9 \\
7.5 \\
8.8 \\
4.5 \\
3.6 \\
3.4\end{array}$ & $\begin{array}{l}0.0 \\
0.0 \\
0.0 \\
0.0 \\
0.0 \\
0.0 \\
0.0 \\
0.0 \\
0.1 \\
0.1 \\
0.0 \\
0.0 \\
0.0 \\
0.0 \\
0.0 \\
0.0 \\
0.1\end{array}$ & $\begin{array}{l}95 \\
97 \\
98 \\
97 \\
98 \\
96 \\
88 \\
69 \\
83 \\
80 \\
46 \\
74 \\
62 \\
73 \\
37 \\
30 \\
28\end{array}$ \\
\hline \multicolumn{5}{|l|}{ Site 289} \\
\hline $\begin{array}{c}1-1,90 \\
3-6,80 \\
4-3,119 \\
6-1,89 \\
8-1,55 \\
10-3,70 \\
12-2,70 \\
14-2,13 \\
16-2,20 \\
18-2,35 \\
20-2,53 \\
22-2,122 \\
24-2,23 \\
26-3,56\end{array}$ & $\begin{array}{r}0.9 \\
27.6 \\
32.7 \\
48.4 \\
67.6 \\
89.2 \\
106.7 \\
125.4 \\
144.3 \\
163.4 \\
182.6 \\
202.2 \\
220.2 \\
241.5\end{array}$ & $\begin{array}{l}10.8 \\
11.0 \\
11.0 \\
10.9 \\
11.1 \\
11.2 \\
11.4 \\
11.3 \\
11.5 \\
11.5 \\
11.6 \\
11.6 \\
11.6 \\
11.4\end{array}$ & $\begin{array}{l}0.1 \\
0.1 \\
0.1 \\
0.1 \\
0.1 \\
0.1 \\
0.1 \\
0.1 \\
0.1 \\
0.1 \\
0.1 \\
0.0 \\
0.0 \\
0.0\end{array}$ & $\begin{array}{l}89 \\
90 \\
91 \\
90 \\
91 \\
93 \\
94 \\
94 \\
95 \\
95 \\
96 \\
96 \\
96 \\
95\end{array}$ \\
\hline
\end{tabular}

TABLE 1 - Continued

\begin{tabular}{|c|c|c|c|c|}
\hline $\begin{array}{c}\text { Sample } \\
\text { (Interval in } \mathrm{cm} \text { ) }\end{array}$ & $\begin{array}{l}\text { Subbottom } \\
\text { Depth } \\
\text { (m) }\end{array}$ & $\begin{array}{l}\text { Total } \\
\text { Carbon } \\
(\%)\end{array}$ & $\begin{array}{l}\text { Organic } \\
\text { Carbon }\end{array}$ & $\mathrm{CaCO}_{3}$ \\
\hline $28-3,63$ & 260.5 & 11.4 & 0.1 & 95 \\
\hline $30-3,108$ & 279.6 & 11.3 & 0.0 & 93 \\
\hline $32-3,64$ & 298.1 & 11.3 & 0.1 & 93 \\
\hline $34-3,59$ & 317.1 & 11.4 & 0.0 & 94 \\
\hline $36-3,63$ & 336.4 & 11.4 & 0.0 & 94 \\
\hline $38-3,62$ & 355.6 & 11.3 & 0.1 & 93 \\
\hline $40-3,52$ & 374.4 & 11.4 & 0.1 & 94 \\
\hline $42-4,67$ & 395.2 & 11.3 & 0.1 & 93 \\
\hline $44-3,17$ & 412.0 & 11.1 & 0.1 & 91 \\
\hline $47-3,27$ & 440.3 & 11.1 & 0.0 & 92 \\
\hline $48-3,43$ & 450.4 & 11.5 & 0.0 & 95 \\
\hline $50-3,67$ & 469.7 & 11.4 & 0.0 & 95 \\
\hline $52-3,128$ & 489.3 & 11.5 & 0.0 & 95 \\
\hline $54-3,100$ & 507.5 & 11.3 & 0.0 & 93 \\
\hline $56-3,83$ & 526.3 & 11.3 & 0.0 & 94 \\
\hline $58-3,86$ & 545.9 & 11.3 & 0.0 & 94 \\
\hline $60-3,73$ & 564.2 & 11.4 & 0.0 & 95 \\
\hline $62-2,52$ & 581.5 & 11.6 & 0.0 & 96 \\
\hline $64-3,58$ & 602.1 & 11.5 & 0.0 & 95 \\
\hline $66-3,104$ & 621.5 & 11.2 & 0.1 & 93 \\
\hline $68-3,62$ & 640.1 & 11.5 & 0.0 & 96 \\
\hline $70-3,55$ & 659.6 & 11.4 & 0.0 & 95 \\
\hline $72-3,71$ & 678.2 & 11.2 & 0.0 & 93 \\
\hline $74-3,37$ & 697.4 & 11.1 & 0.0 & 92 \\
\hline $76-3,79$ & 716.3 & 11.2 & 0.0 & 93 \\
\hline $78-3,77$ & 735.3 & 11.3 & 0.0 & 93 \\
\hline $80-3,80$ & 754.3 & 11.3 & 0.0 & 94 \\
\hline $82-3,47$ & 773.0 & 11.1 & 0.0 & 92 \\
\hline $84-2,23$ & 790.2 & 11.2 & 0.0 & 93 \\
\hline $86-3,67$ & 811.2 & 11.5 & 0.0 & 96 \\
\hline $88-3,38$ & 829.9 & 11.6 & 0.0 & 96 \\
\hline $88-3,63$ & 830.1 & 11.6 & 0.0 & 96 \\
\hline $90-3,60$ & 849.1 & 10.9 & 0.0 & 90 \\
\hline $94-3,70$ & 887.2 & 11.5 & 0.0 & 96 \\
\hline $96-1,101$ & 903.5 & 11.2 & 0.0 & 93 \\
\hline $98-3,135$ & 925.9 & 10.9 & 0.0 & 91 \\
\hline $99-5,87$ & 937.9 & 10.9 & 0.0 & 90 \\
\hline $102-1,93$ & 960.4 & 11.2 & 0.0 & 93 \\
\hline $106-3,103$ & 1001.5 & 11.1 & 0.0 & 92 \\
\hline $108-1,65$ & 1017.2 & 11.4 & 0.0 & 95 \\
\hline $110-1,134$ & 1036.8 & 6.2 & 0.0 & 51 \\
\hline $118-1,106$ & 1112.6 & 12.0 & 0.0 & 100 \\
\hline $124-2,149$ & 1167.5 & 11.9 & 0.0 & 99 \\
\hline $130-7,0$ & 1230.5 & 11.9 & 0.0 & 99 \\
\hline
\end{tabular}

